NUMERICAL ANALYSIS AND MATHEMATICAL MODELLING

BANACH CENTER PUBLICATIONS, VOLUUMI: 24

PWN POLISH SCIISTTITIC PUBLISIJERS

WARSAW 1990

\title{
ОБ АППРОКСИМАЦИИ РЕШЕНИЙ КРАЕВЫХ ЗАДАЧ \\ С ПАРАБОЛИЧЕСКИМ ПОГРАНИЧНЫМ СЛОЕМ
}

\author{
Г. И. ШИШКИН

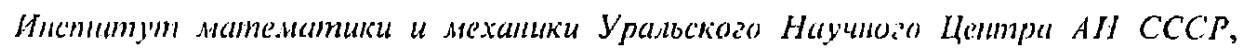 \\ Coepduruck, CCCP
}

Для сингулярно возмущенных краевых задач для уравнений параболического и эллиптического типов с параболическим пограничным слюем предлагается методика построения разностных схем, сходящихся равномерно относительно параметра.

\section{Ввсденис}

Математическое моделирование ряда технологических процессов приводи1 к изучению краевых залач для дифференциалыных уравнений, в которых старшне производныс содержат параметр, принимагоций произвольные эначения из полуинтервала $(0,1]$. При стремлении парамегра к нулю в решеелии таких задач появльюотся осоьбенности типа пограничных и лереходных слоев, что вызывает затруднения при численном их решении [1]. Так, широко распространениые разностные методы решегия [2-4] гребуют применения вблизи границы пага интегрирования, существенно меньшего толицин пограничного слоя. Поэтому предсталяют интерес мегоды построения разностых схем, сходяцихся равномерно отпосительно параметра.

В настояцей работе рассматривается методика построения равномерно по параметру сходнщихся разностных схем для решіния краевых задач для элылиптиеского и параболического уравненй с параболическим пограничным слоем. Для параболического уравнения предлагаегся метод повышения точности (относительно шага временпо́й сетки) раз- 
ностных схем, используюший решения соответствующих разностных задач на последовательности вложенных сегок - экстраполяционный метод Ричардсона (см. $[4,5]$ ).

У решений параболических уравнений, не содержащих производных первого порядка по пространственным переменным, а также эллиптических уравнений, у которых характеристики вырожденного уравнения параллельны границе области, при стремлении параметра к нулю возникают пограничные слои, описываемые уравнениями параболического типа (параболические пограничные слои). В окрестности параболического пограничного слоя решение задачи и его производные по направлению вдоль границы области ограничены равномерно по параметру, производные по нормали к границе, неограниченно возрастают при стремлении параметра к нулю. В этой окрестности исходное дифференциальное уравнение в тастных производных можно рассматривать относительно переменной, ортогональной границе области как обыкновенное дифференциальное уравнение со специальным свободным цленом (содержащим производные по направлению, касательному к границе). Такое поведение решения в окрестности параболического слоя позволяет строить разностные схемы для уравнений в частных производных на основе разностных схем для сингулярно возмущенных краевых задач для обыкновенных дифференциальных уравнений. Для аппроксимации производных, ортогональных границе области, используются соответствуюцие разностные производныс на специальным образом сгущающихся сетках. Закон сгущения сетки такой же как и в случае обыкновенного ди()ференциального уравнения [2].

В $\S 1,3$ рассматривается построение разностных схем для решения краевых задач для уравнений параболического и эллиптического типов, приводятся разностные схемы и теоремы о равномерной по параметру сходимости этих схем. В случае эллиптического уравнения в окрестности экспоненцтального пограничного слоя для аппроксимации дифференциального оператора, содержацего производные по направлению вдоль границы области, используется разностный оператор схемы пгодгонки (для обыкновенного дифференциального уравнения) [6]. В § 2 для параболитеского уравнения по методу Ричардсона строятся приближенные решения более высокого порядка точности (по временной перемегшой). Развернутое обоснование приведеных здесь результатов и их обобшегия даются в работах [8-10].

Отметим, что в [5] используется метод Ричардсона для решения уравнений параболического типа в том случае, когда уравнения не содержат малый параметр, а для решения задачи применяются равномерные сетки, у которых паг сетки по временно́й переменной пропорционален квадрату шага сетки по иространственной переменной. 


\section{§ 1. Разиостная схема для параболического уравнения}

1. В области $D=\{(x, t) / 0<x<1,0<t \leqslant T\}$ с границей $\Gamma=\bar{D} \backslash D$ рассмотрим задачу Дирихле для параболического дифференциального уравнения

$$
\begin{gathered}
\left\{\varepsilon \frac{\partial}{\partial x}\left(a(x, t) \frac{\partial}{\partial x}\right)-c(x, t)-p(x, t) \frac{\partial}{\partial t}\right\}=-\psi(x, t), \quad(x, t) \in D \\
u=\varphi(x, t), \quad(x, t) \in \Gamma
\end{gathered}
$$

где $a(x, t), c(x, t), p(x, t), \psi(x, t),(x, t) \in \bar{D}$, и $\varphi(x, t),(x, t) \in \Gamma$ - достаточно гладкие функции, причем $a(x, t), c(x, t), p(x, t) \geqslant \alpha_{0}>0,(x, t) \in \bar{D}$; $\varepsilon \in(0,1]$. При стремлении параметра к нулю в окресности боковой границы области $D$ возникает пограничный слой - параболический пограничный слой, описываемый уравнением параболического типа. Для задачи (1.1), (1.2) построим разностную схему, сходящуюся равномерно относительно параметра.

Производная по времени решения задачи ограничена равномерно по параметру. При фиксированном значении $t \in(0, T]$ уравнение (1.1) относительно переменной $x$ можно рассматривать как обыкновенное дифференциальное уравнение с правой частью $\Psi(x, t)=-\psi(x, t)+p(x, t) \times$ $x \partial u(x, t) / \partial t$. Поставим ему в соответствие трехточечную разностную схему первого порядка точности, причем производную $\partial u / \partial t$, входящую в правую часть, будем аппрокскмировать разностной производной. Эту схему строим интегро-интерполяционным методом $[3,4]$.

2. В области $D$ введем сетку $D_{h \tau}$, полагая $\bar{D}_{h \tau}=\bar{\omega}_{h} \times \bar{\omega}_{\tau}, D_{h \tau}=$ $=\bar{D}_{h \tau} \cap D, \quad$ где $\quad \bar{\omega}_{\tau}=\left\{t_{k} / t_{k}=k \tau, k=0,1, \ldots, K\right\}, K \tau=T, \omega_{h}=\left\{x_{i} / i=\right.$ $\left.=0,1, \ldots, N, x_{0}=0, x_{N}=1\right\}, \Gamma_{h \tau}=\bar{D}_{h \tau} \cap \Gamma$. Будем обозначать $f\left(x, t_{k}\right)=$ $=f_{k}(x), f_{k}\left(x_{i}\right)=f_{k}^{i}, h=\max _{i} h_{i}, h_{i}=x_{i+1}-x_{i}$.

Выпишем разностную схему для задачи (1.1), (1.2):

$$
\begin{gathered}
\varepsilon\left(a_{k}^{h, i-1} z_{\bar{x}}\right)_{\bar{x}}-c_{k}^{i} z-p_{k}^{i} z_{\bar{t}}=-\psi_{k}^{i},(x, t) \in D_{h r}, \\
z=\varphi_{k}^{i}, \quad(x, t)=\left(x_{i}, t_{k}\right) \in \Gamma_{h t},
\end{gathered}
$$

где

$$
a_{k}^{h, i}=2 a_{k}^{i} a_{k}^{i+1}\left(a_{k}^{i}+a_{k}^{i+1}\right)^{-1} .
$$

Эта схема является мопотонной при любых $h_{i}, \tau, \varepsilon$, (см. [3, гл. VII, $§ 1])$.

Заметим, что на произвольных сетках, максимальный шаг которых стремится к нулю, разностная схема (1.3)-(1.5) сходится при фикскрованном значении параметра и не сходится равномерно относительно параметра. 
3. Вместе с сеткой $\omega_{1}$ с произвольным расположением узлов будем рассматривать такие сетки $\omega_{h}^{n}$ со сгущаюцимися узлами вблизи концов отрезка $[0,1]$, для которых выполняются соогношения

$$
\max _{1 \leqslant i \leqslant N-1} \delta_{i}^{n} \leqslant M h^{n}, \quad m N^{-1} \leqslant h \leqslant M N^{-1},
$$

где $\delta_{i}^{n}=h_{i}^{n}+h_{i-1}^{n}+\min \left(h_{i}^{n} \varepsilon^{-n / 2} \eta_{h}^{i}+h_{i-1}^{n} \varepsilon^{-n / 2} \eta_{h}^{i-1} ; \eta_{h}^{i}+\eta_{h}^{i-1}\right), i=1,2, \ldots, N-1$, $\eta_{t r}^{i}=\exp \left(-m_{1} x_{i} / \varepsilon^{1 / 2}\right)+\exp \left(-m_{1}\left(1-x_{i+1}\right) / \varepsilon^{1 / 2}\right), m_{1}<m_{0}, n>0-$ целое число.

Через $M(m)$ будем обозиачать достаточно большие (достаточно малые) положительные постояпше, не занияяцие от $\varepsilon, h_{i}, \tau$ и $T$, где $\left.\varepsilon \in(0,1], h_{i}, \tau<1, T \in(0, \infty)\right)$.

Построим сетку $\omega_{h}^{n}$, подобную приведенной в [2]. Пусть $\lambda_{i}=$ $=M_{0} \varepsilon^{1 / 2} \ln \left(1-i / N_{0}\right)^{-1}$, где $N_{0}=h^{-1}, N_{0}$ - целое число, а $R-$ нанбольший номер $i$, для которого выполняются неравенства: $\lambda_{i}-\lambda_{i-1} \leqslant h$, $\lambda_{i} \leqslant 1 / 2$. Пусть узлы сетки раснолагантея симметрично относительно середины отрезка $[0,1]: x_{i}=1-x_{N-1}, i=0,1, \ldots, N, x_{N}=1$, причем середина отрезка $[0,1]$ гриндлежит сетке. Положим $x_{i}=\lambda_{i}$ при $i \leqslant R$ и $x_{i}=x_{R}+(i-R) h$ при $x_{R}<1 / 2$. Нетрудио видеть, что $(N-1)^{-1} \leqslant$ $\leqslant h \leqslant 3(N-1)^{-1}$. Используя технику оценок работы [2] (примененную в оценке погреппости решения), можно показать справедливость неравенства

$$
\max _{i} \delta_{i}^{n} \leqslant M h^{n}, \quad i=0,1, \ldots, N-1 \text { при } m_{1} M_{0} \geqslant n .
$$

Таким образом, построена сетка $\omega_{h}^{\prime \prime}$.

Будем рассматривать, сетки. узыы которых в огрестности боковой грапицы области $\bar{D}$ сгупағося по пространственной переменной. Пусть

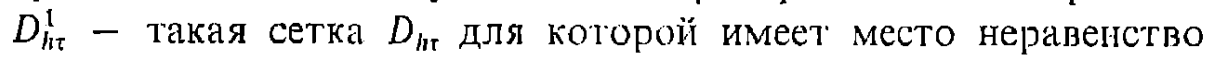

$$
\max _{i . k} \sigma_{i k} \leqslant M h, \quad\left(x_{i}, l_{k}\right) \in D_{h t}
$$

$\sigma_{i k}$ при $t=t_{k}$ есть $\delta_{i}^{1}$, где $\eta_{h}^{i}=\eta_{h}^{i}\left(m_{1}\right), m_{1}<m_{0}, m_{0}=\min \left[c\left(x, t_{k}\right) / a\left(x, t_{k}\right)\right]^{1 / 2}$. Так, сетка $\omega_{h}^{1} \times \omega_{\tau}$, где $\omega_{h}^{1}-$ сетка на отрезке $[0,1]$, построеннал с использованием функцин

$$
\lambda_{i}=M_{0} c^{1 / 2} \ln \left(1-i / N_{0}\right)^{-1} \quad \text { при } \quad m_{1} M_{0} \geqslant 1, \quad m_{1}<\underset{b}{\min }[c(x, l) / a(x, t)]^{1 / 2}
$$

есть сетка $D_{h r}^{1}$.

Будем обозначать $f(x, t) \in H^{l+k, l / 2}(\bar{D})$, если $\imath^{k} f(x, t) / \partial x^{k} \in H^{l, t / 2}(\bar{D})$, $k \geqslant 0$ - целое число (см. [7]), $H^{l .1 / 2}(\bar{D})=H^{(l)}(\bar{D})$.

Справедлива́ следуюліны

TeOpema 1. $\Pi_{y} c m b, a(x, l) \in H^{t+5 . / / 2}(\bar{D}), c(x, t), p(x, t) \in H^{t+4 . t / 2}(\bar{D}), l>0$.

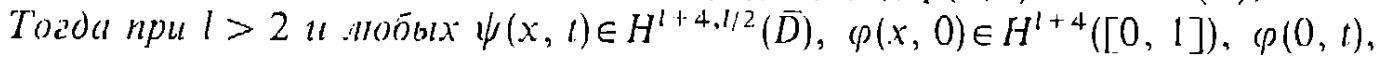


$\varphi(1, t) \in H^{1+l / 2}([0, T])$, удовлетворяюиих условит согласования порядка $[l / 2]+1$, функиия z (решение задачи (1.3)-(1.5) на сетке $D_{h \tau}^{1}$ ) при $h, \tau \rightarrow 0$ сходится к решению задачи (1.1), (1.2) со скоростыо $O(h+\tau)$ равномерно относительно параметра и величины интервала Т. Для решения задачи (1.3)-(1.5) на с'етках $\bar{D}_{h \tau}, \bar{D}_{k \tau}^{1}$ при любых $h$ и $\tau$ справедливы оченки

$$
\begin{gathered}
|u-z| \leqslant M(h+\tau), \quad(x, t) \in \bar{D}_{h \tau}^{1}, \\
|u-z| \leqslant M\left(\max _{i, k} \sigma_{i k}+\tau\right), \quad(x, t) \in \bar{D}_{h \tau} .
\end{gathered}
$$

\section{§ 2. Замечаикя и обобщения}

1. Схема (1.3), (1.4) остается монотонной, если положить

$$
a_{k}^{h, i}=\left(a_{k}^{i}+a_{k}^{i+1}\right) / 2
$$

либо использовать другие формулы (см., например, [3, гл. VII, $\S 1$, п. 10]), аппроксимирующие $a(x, t)$ на множестве $x_{i} \leqslant x \leqslant x_{i+1}, t_{k-1} \leqslant$ $\leqslant t \leqslant i_{k}$, при условии, что $a_{k}^{h, i}>0$ при $\left(x_{i}, t_{k}\right) \in \bar{D}_{h \tau}$.

Для решения задачи $(1.3),(1.4)$ выполняется утнерждение теоремы 1 , если $a_{k}^{h, i}$ аппроксимирует $a\left(\left(x_{i}+x_{i+1}\right) / 2, t_{k}\right), i=0,1, \ldots, N-1, t_{k} \in \omega_{\tau}$ с точностыю $O\left(h_{i}^{2}\right)$.

2. При использовании формул (1.5) или (2.1) для решения задачи (1.3), (1.4) на сетке $\bar{D}_{h \tau}=\bar{\omega}_{h}^{2} \times \bar{Q}_{\mathrm{r}}$ выполняется оценка $|u-z| \leqslant M\left(h^{2}+\tau\right)$, $\left(x_{i}, t_{k}\right) \in \bar{D}_{h t} ; \omega_{l i}^{2}=\omega_{h}$ из работы [2].

3. Если применяется сетка с переменным шагом интегрирования по $t$, то в оцегках (1.6), (1.7)

$$
\tau=\max _{k} \tau_{k}, \quad \tau_{k}=t_{k+1}-t_{k},
$$

$t_{k}-$ точки разбиения интервала $[0, T]$.

4. Пусть $u(x, t)$ - решение уравнения (1.1), удовлетворяющее краевым условиям третьего рода

$$
\begin{gathered}
-\alpha_{01}(t) \varepsilon^{1 / 2}-\frac{\partial u(0, t)}{\partial x}+\alpha_{00}(t) u(0, t)=\varphi_{0}(t), \\
\alpha_{11}(t) \varepsilon^{1 / 2} \frac{\partial u(1, t)}{\partial x}+\alpha_{10}(t) u(1, t)=\varphi_{1}(t),
\end{gathered}
$$

где $\alpha_{01}(t), \alpha_{11}(t) \geqslant \alpha>0, \alpha_{00}(t), \alpha_{10}(t)>0, t \in[0, T]$. Этой задаче поставим в соответствие разностную схему: во внутренних узлах сетки $\bar{D}_{h \tau}-$ 
уравнения (1.3), а в граничных узлах - уравнения

$$
\left\{\begin{array}{l}
-\alpha_{01 k} \varepsilon^{1 / 2}\left(a_{k}^{0}\right)^{-1} A_{k}^{0}(\psi, z)+\alpha_{00 k} z_{k}^{0}=\varphi_{0 k}, \\
\alpha_{11 k} \varepsilon^{1 / 2}\left(a_{k}^{N}\right)^{-1} A_{k}^{N-1}(\psi, z)+\alpha_{10 k} z^{0}=\varphi_{1 k}, \\
k=1,2, \ldots, K ; \quad z_{0}^{i}=\varphi_{0}^{i}, \quad i=0,1, \ldots, N,
\end{array}\right.
$$

где $A_{k}^{0}(\psi, z)=a_{k}^{h, 0}\left[z_{k x}^{0}-\varepsilon^{-1} h_{0}\left(z_{k}^{0}-\varphi_{k}^{0}\right) /\left(2 a_{k}^{0}\right)\right], \quad A_{k}^{N-1}(\psi, z)=a_{k}^{h, N-1}\left[z_{k x}^{N}+\right.$ $\left.+\varepsilon^{-1} h_{N-1}\left(z_{k}^{N}-\psi \psi_{k}^{N}\right) /\left(2 a_{k}^{N}\right)\right]$.

Если $\quad a \in H^{l+5, l / 2}(\bar{D}), c, p, \psi \in H^{l+4, l / 2}(\bar{D}), \alpha_{00}, \alpha_{01}, \alpha_{10}, \alpha_{11}, \varphi_{0}, p_{1} \in$ $\in H^{(l+1) / 2}([0, T]), \varphi \in H^{l+4}([0,1]), l>2$, и выполнены условия согласования порядка $[(l+1) / 2]$, то для решения задачи (I.3), (I.5), (2.2) сохраняется утверждение теоремы I.

5. Пусть коэффициент $a$ не зависит от $t, a \in H^{l+3}([0,1]), c, p \in$ $\in H^{l+2, l / 2}(\bar{D})$. Тогда при $l>4$ и любых $\psi \in H^{l+2 . l / 2}(\bar{D}), \varphi(x, 0) \in H^{l+2}([0,1])$, $\varphi(0, t), \varphi(1, t) \in H^{1+l / 2}([0, T])$, удовлетворяюших условию согласования порядка $[l / 2]+1$, выполняется утверждение теоремы 1. В этом случае при $\left(x_{i}, t_{k}\right) \in \overline{D_{h t}^{1}}$ для решения задачи (1.3)-(1.5) выполняются также оценки

$$
\begin{gathered}
\left|\frac{\partial u\left(x_{i}, t_{k}\right)}{\partial t}-z_{t_{i}}^{i}\right| \leqslant M(h+\tau), \quad k \neq 0, \\
\varepsilon^{1 / 2}\left|\frac{\partial u\left(x_{i}, t_{k}\right)}{\partial t}-z_{k x}^{i}\right| \leqslant M(h+\tau), \quad i-1 \leqslant j \leqslant i, j \neq N, \\
\varepsilon\left|\frac{\partial^{2} u\left(x_{i}, t_{k}\right)}{\partial x^{2}}-z k_{\dot{x} x}\right| \leqslant M(h+\tau), \quad|i-j| \leqslant 1, j \neq 0, N .
\end{gathered}
$$

При выводе этих оценӧк используются уравнения, полученные применением оператора первой разностной производной по $t$ к уравнениям (1.1), (1.3).

6. Пусть $a(x, t) \in H^{(l+1)}(\bar{D}), c(x, t), p(x, t) \in H^{(l)}(\bar{D}), l>0$ - нецелое число. Если функции $\varphi$ и $\psi$ в точках $(0,0),(0,1)$ удовлетворяют соотношениям

$$
\frac{\partial^{k}}{\partial x^{k}} \varphi, \frac{\partial^{n}}{\partial t^{n}} \varphi=0, \quad k, 2 n \leqslant[l]+2, \quad \frac{\partial^{k+n}}{\partial x^{k} \partial t^{n}} \psi=0, \quad k+2 n \leqslant[l],
$$

то для задачи (1.1), (1.2) выполнены условия согласования порядка $[l / 2]+1$. Построим приближенные решения пошышенной точности, используя решения разностных задач (1.3), (1.4) на неравномерных сетках.

Пусть $z^{(j)}$ - решение задачи (1.3), (1.4) при $D_{h \tau}=D\left(h, \tau^{(j)}\right) D\left(h, \tau^{(j)}\right)=$ $=\omega_{h} \times \omega\left(\tau^{(j)}\right), \omega_{h}-$ неравномерная сетка на отрезке $[0,1], \omega\left(\tau^{(j)}\right)=\omega_{\tau}$ при $\tau=\tau^{(j)}, j=0,1, \ldots, p$; 
$p$ - целое число. Пусть $\left|\tau^{(j)}\right| \tau^{(j+1)}-1 \mid \geqslant \alpha, \alpha>0$. На пересечении сеток

$$
\bigcap_{0 \leqslant j \leqslant p} D\left(h, \tau^{(j)}\right)=D_{1}(p)
$$

(пусть $D_{1}(p) \neq \varnothing$ ) определим функцию $Z^{p}$ :

$$
Z^{p}(x, t)=\sum_{j=0}^{p} \gamma^{(j)} z^{(j)}(x, t), \quad(x, t) \in D_{1}(p)
$$

где $\gamma^{(j)}$ - решение системы

$$
\sum_{s=0}^{p} \gamma^{(s)}=1, \quad \sum_{s=0}^{p} \gamma^{(s)}\left(\tau^{(s)}\right)^{n}=0, \quad n=1,2, \ldots, p .
$$

Через $\omega_{h}^{\alpha}$ обозначим такую сетку $\omega_{h}$ со сгущающимися вблизи концов отрезка $[0,1]$ узлами, для которой выполняются соотношения

$$
\max _{1 \leqslant j \leqslant N-1} \varrho_{j}(\alpha) \leqslant M h^{\alpha} \quad \text { при } \quad 0<\alpha \leqslant 2, \quad h \leqslant M N^{-1},
$$

где

$$
\varrho_{j}(\alpha)=\left\{\begin{array}{rr}
\left(h_{j}+h_{j+1}\right)^{\alpha}+\min \left\{\left(h_{j} \varepsilon^{-1 / 2}\right)^{\alpha} \eta_{j}^{h}+\left(h_{j+1} \varepsilon^{-1 / 2}\right)^{\alpha} \eta_{j+1}^{h},\right. & \\
\left.\eta_{j}^{h}+\eta_{j+1}^{h}\right\}, & \alpha \in(0,1], \\
\left(h_{j}+h_{j+1}\right)^{\alpha}+\varepsilon\left|h_{j+1}-h_{j}\right|+\min \left\{\left(h_{j} \varepsilon^{-1 / 2}\right)^{\alpha} \eta_{j}^{h}\right. & \\
\left.+\left(h_{j+1} \varepsilon^{-1 / 2}\right)^{\alpha} \eta_{j+1}^{h}+\left|h_{j+1}-h_{j}\right| \varepsilon^{-1 / 2} \eta_{j}, \eta_{j}^{h}+\eta_{j+1}^{h}\right\}, & \alpha \in(1,2] .
\end{array}\right.
$$

Определим $v(q)$, где $q$ - произвольное неотрицательное число, полагая $v(q)=q-1$, если $q-$ целое положительное число, $v(q)=[q]-l-$ в противном случае $\left([q]\right.$ - целая часть числа). Положим $D_{2}^{\alpha}(p)=$ $\bigcap_{0 \leqslant j \leqslant p} D\left(h, \tau^{(j)}\right)$, где $D\left(h, \tau^{(j)}\right)=\omega_{h}^{\alpha} \times \omega\left(\tau^{(j)}\right)$.

Справедлива следующая

Tеотема 2. Пусть $l>0$ - нечелое число, коэффичиенты $c(x, t), p(x, t) \in$ $\in H^{r, s}(\bar{D}), a(x, t) \in H^{r-1, s}(\bar{D})$, zде $r=l+2[l / 2]+4, s=l / 2$, причем $\psi(x, t) \in$ $\in H^{r, s}(\bar{D}), \varphi(x, 0) \in H^{r}([0,1]), \varphi(x, t) \in H^{s+1}([0, T]), x=0,1$, удовлетворяіот условиям (2.3). Тогда фуннкия $Z^{p}(x, t), 0 \leqslant p \leqslant[l / 2]$, при $h, \tau \rightarrow 0(N=$ $=N(h) \rightarrow \infty)$ сходится равномерно относительно параметра є. Для функции $Z^{p}$ при $h, \tau<1, \varepsilon \in(0,1]$ справедлива оченка

$$
\left|u(x, t)-Z^{p}(x, t)\right| \leqslant M\left(h^{\lambda_{1}}+\tau^{\lambda_{2}}\right), \quad(x, t) \in D_{2}^{\alpha}(p),
$$

где $\lambda_{1}=\min \left(\alpha, \vartheta\left(\lambda_{2}, l\right)\right), 0 \leqslant \lambda_{2} \leqslant \min (p+1, l / 2), \vartheta\left(\lambda_{2}, l\right)=\min \left[2, l-\nu\left(2 \lambda_{2}\right)\right]$.

Пусть, например, $\alpha=2, l>2$, и $p=[l / 2]-1$. В этом случае приближенное решение $Z^{p}(x, t),(x, t) \in D_{2}^{2}(p)$ при $h, t \rightarrow 0$ сходится равномерно относительно параметра $\varepsilon \in(0,1]$ со скоростью $O\left(h^{2}+\tau^{\lambda}\right), \lambda=l / 2-1$. 


\section{§ 3. Разиостная схема для эллиптического уравнения}

1. В прямоугольнике $D=\left\{x \mid 0<x_{i}<d_{i}=1,2\right\}$ с граннцей $\Gamma=\bar{D} \backslash D$ рассмотрим задачу Дирихле

$$
\begin{gathered}
\left\{c \sum_{i=1,2} a_{i}(x) \frac{\partial^{2}}{\partial x_{i}^{2}}+b(x) \frac{\partial}{\partial x_{2}}-c(x)\right\} u=f(x), \quad x \in D, \\
u=\varphi(x), \quad x \in \Gamma .
\end{gathered}
$$

3десь $a_{i}(x), b(x), f(x) c(x), x \in \bar{D}, \varphi(x), x \in \Gamma$ - достаточно гладкие фунгция, причем $a_{i}(x), b(x) \geqslant \alpha>0, c(x) \geqslant 0, x \in \bar{D}$.

При стремлении параметра к нулю в окрестности боковых сторон прямоугольника $D$ возникают параболические пограничные слои, а в окрестности верхней стороны - экспоненциальный пограничный слой. В окрестности параболического пограничного слоя пронзводные по $x_{2}$ являются ограниченными равномерно по $\varepsilon$, а произведение параметра на производную второго порядка $\partial^{2} u / \partial x_{2}^{2}$ стремится к нулю при $\varepsilon \rightarrow 0$. Поэтому главный член сингулярной части решения в параболическом пограничном слое описывается параболическим уравнением. Разностную схему для аппроксимации регшения в окрестности боковых границ прямоугольника удобно строить, используя схему на сгущаюшейся сетке для параболического уравнения.

Производные ренения задачи по $x_{1}$ в окрестности экспонепциального пгограничного слоя ограничены равномерно по є. Уравнение (3.1) в этой окрестности можно рассматривать как обыкновешне лифференциальное уравнение относительно переменгой $x_{2}$. Для апроксимации репения воспользуемся разностной схемой (метода экспоненциальной шодгонки) для обыкновенного дифференциального урдвнения. Производные по $x_{1}$ аппроксимируем разностными производными на неравномерной сетке.

2. На множестве $\bar{D}$ введем сетку $\bar{D}_{h}=\bar{\omega}_{h 1} \times \bar{\omega}_{h 2}$, где

$$
\begin{aligned}
\vec{\omega}_{h 1}=\left\{x_{1}^{i} \mid i=0,1, \ldots, N_{1}, x_{1}^{0}=0, x_{1}^{N_{1}}=d_{1}\right\}, \\
\bar{\omega}_{h 2}=\left\{x_{2}^{j}=j h_{2}^{*}, j=0,1 \ldots, N_{2}, x_{2}^{0}=0, x_{2}^{N_{2}}=d_{2}\right\} .
\end{aligned}
$$

Положим $\Gamma_{h}=\bar{D}_{h} \cap \Gamma, D_{h}=\bar{D}_{h} \cap D$. Задаче (3.1). (3.2) conociaвим разиостную схему

$$
\begin{aligned}
& \varepsilon a_{1}(x) z_{\bar{x}_{1} \hat{x}_{1}}+\gamma(x) z_{x_{2} \bar{x}_{2}}+b(x) z_{\bar{x}_{2}}-c(x) z=f(x), \\
& \quad x \in D_{h} ; z=\varphi(x), \quad x \in \Gamma_{h} .
\end{aligned}
$$

Здесь $\gamma(x)=2^{-1} h_{2}^{*} b(x) \operatorname{cl} \gamma(x), \psi(x)=h_{2}^{*} h(x)\left[2 \varepsilon_{1} u_{2}(x)\right]^{-1}$. Схема (3.3) является монотонной. На сетке $\bar{D}_{h}^{1}=\bar{\omega}_{h 1} \times \bar{\omega}_{h 2}$, где $\bar{\omega}_{h 1} \equiv \bar{\omega}_{h 1}^{1}$, равномерную по параметру сходимость схемы устанавливает 
TEOPEMA 3. IIycmb $a_{1}(x), a_{2}(x), b(x), c(x), f(x) \in C^{3}(\bar{D}), u(x) \in C^{4}(\bar{D})$. Пусть, короме того, бункии $f(x), \varphi(x)$ в угловых точках $\left(0, d_{2}\right),\left(d_{1}, d_{2}\right)$ yдовлетвор siот условию: $b(x) \partial \varphi(x) / \partial x_{2}-c(x) \varphi(x)-f(x)=0$. Тогда разностная схема́ (3.3) при $h \rightarrow 0$ сходится в прямоугольнике $\bar{D}$ со скоростью $O\left(h^{1 / 4}\right)$ равномерно относительно параметра

$$
|u-z| \leqslant M h^{1 / 4}, \quad x \in \bar{D}_{h}^{1} .
$$

Заметим, что при фиксированном значении параметра разностная схема (3.3) на сетке $\bar{D}_{h}$ сходится со скоростью $O(h)$, а на сетке $D_{h}^{2}=$ $=\omega_{h_{1}} \times \omega_{h_{2}}$, где $\omega_{h_{1}} \equiv \omega_{h_{r}}^{2}$, со 'скоростью $O\left(h^{2}\right)$.

\section{Литература}

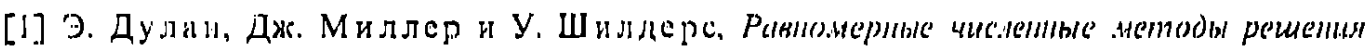

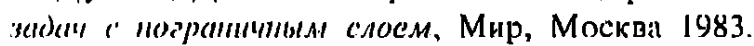

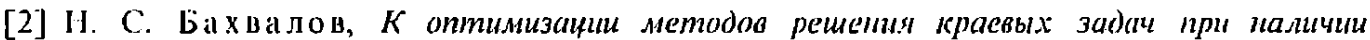

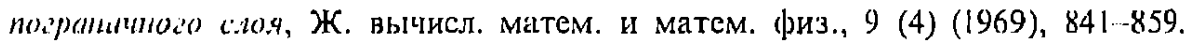

[3] А. А. Самарокий, Теприя paзиостиых схем, Наука, Москва 1977.

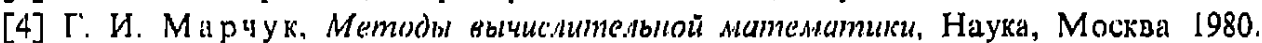

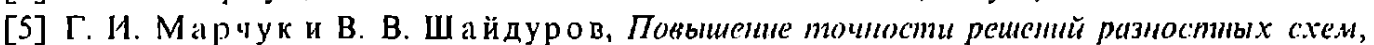
Наука, Москва 1979.

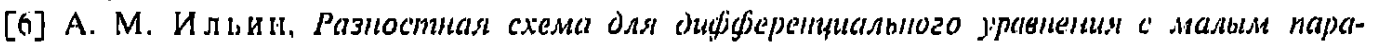
мепроли при старией производиой, Матем. заметки, вып. 2 1969, 237-248.

[7] О. А. Ладыженская, В. А. Солоншиков и Н. Н. У ральцева, Липейлье икоазили-

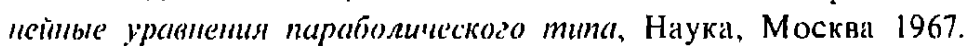

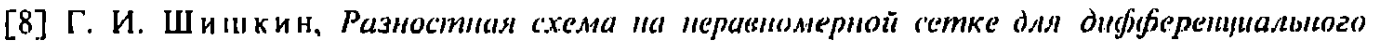

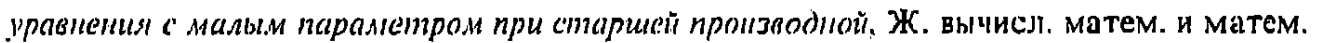
()нз., 23 (3) (1983), 609-619.

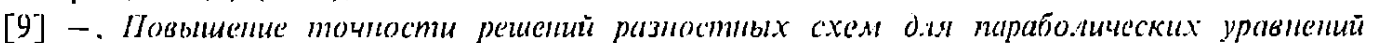

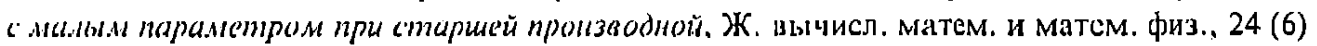
(1984), 864-875.

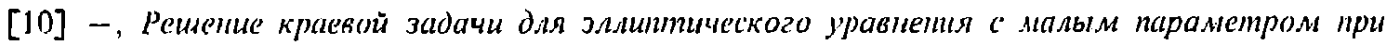
('maрииах производиых, ЖК. вынисл. матем. и матем. физ., 26 (7) (1986), 1019-1031.

Presented to the Semester

Numerical Analysis and Mathematical Modelling

February 25-May 29, 1987 